\title{
Improved RSSI Positioning Algorithm for Coal Mine Underground Locomotive
}

\author{
Bin Ge, ${ }^{1}$ Kai Wang, ${ }^{1}$ Jianghong Han, ${ }^{2}$ and Bao Zhao ${ }^{1}$ \\ ${ }^{1}$ School of Computer Science and Engineering, Anhui University of Science \& Technology, Huainan, Anhui 232001, China \\ ${ }^{2}$ School of Computer and Information, Hefei University of Technology, Hefei 230009, China \\ Correspondence should be addressed to Bin Ge; bge@aust.edu.cn
}

Received 8 December 2014; Accepted 23 January 2015

Academic Editor: Peter Jung

Copyright (c) 2015 Bin Ge et al. This is an open access article distributed under the Creative Commons Attribution License, which permits unrestricted use, distribution, and reproduction in any medium, provided the original work is properly cited.

\begin{abstract}
Aiming at the large positioning errors of traditional coal mine underground locomotive, an improved received signal strength indication (RSSI) positioning algorithm for coal mine underground locomotive was proposed. The RSSI value fluctuates heavily due to the poor environment of coal mine underground. The nodes with larger RSSI value corrected by Gaussian-weighted model were selected as beacon nodes. In order to reduce the positioning error further, the estimated positions of the locomotives were corrected by the weighted distance correction method. The difference between actual position and estimated position of beacon node was regarded as the positioning error and was given a corresponding weight. The results of simulation show that the positioning accuracy of Gaussian-weighted model is better than statistical average model and Gaussian model and it has a high positioning accuracy after correcting positioning error correction. In the $10 \mathrm{~m}$ of communication range, positioning error can be maintained at $0.5 \mathrm{~m}$.
\end{abstract}

\section{Introduction}

Due to the closed and harsh environment of coal mine and the narrow and intricate roadway, mine transportation accidents often occur. It always accounts for a large proportion in all kinds of coal mine accidents. Achieving coal mine underground locomotive positioning technology is significant for reducing economic losses and transportation accidents. It also not only can allocate resource reasonable, but also can repair locomotive timely when it breaks down.

At present, the underground positioning technologies are Bedford and Kennedy [1] proposed evaluation of ZigBee (IEEE 802.15.4) Time-of-Flight-based distance measurement for application in emergency underground navigation. Lei et al. [2] introduced a positioning technology of underground moving target which is based on Wi-Fi (wireless-fidelity) and Web GIS (geographic information system). It uses trilateration algorithm to calculate relative coordinate of the target. Hongpeng Chi et al. [3] introduced a laser-based positioning system. The laser light is reflected back to locomotive using time difference to calculate the distance and achieve positioning. Gao et al. [4] proposed a locomotive position system in coal mine which is based on the piezoelectric accelerometer, through integrating over the collected acceleration value of the electric locomotive and solving out the speed and position. Zhang et al. [5] used the inertial navigation system into the coal mine locomotive localization system. Han et al. [6] introduced a weighted centroid localization algorithm which is based on received signal strength indicator. It can access to the path of decline index dynamically, and then calculate its own location using weighted centroid algorithm.

These researches of the positioning technology provide some useful exploration for achieving the coal mine locomotive positioning, but they have defect in practical application. In literature $[1,2]$ they are simple but have low accuracy and do not suit high-precision mine operations. In literature [3] it is influenced by the environment and may get larger positioning errors in harsh environment. In literatures $[4,5]$ they have limits; for example, if locomotive gets rapidly speedy, positioning accuracy will become low and there is greater cumulative error and it takes longer time in positioning process. In literature [6] it is achieved easily and has better accuracy, but it is not stable and the error fluctuates greatly with the change of locomotive location. 
According to the analysis, considering the low cost, complexity, and without any additional hardware device of RSSI location and arranging nodes sufficiently in the pit [79], this paper uses RSSI location and makes improvement. The positioning errors of RSSI positioning algorithm for coal mine underground locomotive have two primary factors. Firstly, the RSSI value is very unstable, because it is influenced by bad environment such as diffraction, multipath, and obstructions. Secondly, location algorithm is not precise enough and cannot deal with some inaccurate date effectively. The main methods of managing RSSI value are statistical mean model, weighted model, Gaussian model, and so forth. Literature [10] simulated several models and the results show that the highest ranging precision is Gaussian model. Literature [11] proposed a correction algorithm based on Gaussian anchor nodes; literature [12] proposed a D-Gaussian model, it can filtrate variance level interference. Literature [13] used hybrid filtering method to optimize RSSI value. This paper presents a Gaussian-weighted model to get more accurate distance, uses weighted least squares method to estimate location of the locomotive, and corrects positioning error using weighted distance correction method.

\section{Wireless Communication Model and RSSI Ranging Model}

2.1. Wireless Communication Model of Coal Mine. Because coal mine tunnel is narrow and has rails, the nodes cannot be arranged anywhere. In order to collect wireless signal of the nodes correctly, the beacon nodes are arranged on both sides of the track and spaced in a certain distance to reduce interference between the signals, as shown in Figure 1.

In Figure 1, Wireless AP (access point) is a node which can communicate with other wireless AP using wireless technology and A, B, C, and D are the beacon nodes which are arranged on both sides of the rail. When the locomotive is running to this location shown in Figure 1, it can communicate with beacon nodes and it is ensured that the number of the nodes which can communicate with locomotive is greater than or equal to 4 . That ensures the calculation of exact location. In order to guarantee the number of communication nodes, the distance between nodes $\mathrm{A}$ and $\mathrm{B}$ should not exceed the range of the communication; the same goes for C and D, in Figure 1. To obtain more accurate RSSI values, the distance between $\mathrm{A}$ and $\mathrm{B}$ or $\mathrm{C}$ and $\mathrm{D}$ can be reduced. If the distance is short sufficiently, the number of communication nodes may be increased to five or more and the relative cost and complexity of the algorithm will increase.

2.2. RSSI Ranging Model. As the propagation distance of radio signal increase, the signal strength decay. It has different attenuation amplitude, in different environments. In WSN (Wireless Sensor Network), the commonly used wireless signal propagation models are: free space propagation model, log-distance path loss model, Hata model, log-distance distribution model, and so forth $[14,15]$.
Log-distance distribution model can be used to calculate the path loss when nodes received information. The model is shown as follows:

$$
\mathrm{PL}(d)=\mathrm{PL}\left(d_{0}\right)+10 \times n \times \lg \left(\frac{d}{d_{0}}\right)+X_{\sigma},
$$

where $\mathrm{PL}(d)$ is the path loss after signal transmitting distance $d$, the unit of the path loss is $\mathrm{dB}, X_{\sigma}$ is a Gaussian distribution random variable whose mean value is 0 and standard deviation is $\sigma$ (generally $4-10$ ), $n$ is path attenuation factor and always takes $2-5, d_{0}$ is reference distance and usually equals 1 meter, and $\operatorname{PL}\left(d_{0}\right)$ is a path loss after signal transmitting distance $d_{0}$ (generally $1 \mathrm{~m}$ ).

The RSSI of nodes at distance $d$ is

$$
\text { RSSI }=P_{\text {send }}+P_{\text {amplify }}-\mathrm{PL}(d),
$$

where $P_{\text {send }}$ is transmitting power and $P_{\text {amplify }}$ is the gain of antenna.

Similarly

$$
A=P_{\text {send }}+P_{\text {amplify }}-\operatorname{PL}\left(d_{0}\right),
$$

where $A$ is the RSSI at the distance of $d_{0}$.

By (1), (2), and (3) the distance between unknown nodes and beacon nodes can be calculated:

$$
d=10^{\left(\left(A-\mathrm{RSSI}-X_{\sigma}\right) / 10 n\right)} d_{0}
$$

\section{Improved RSSI Positioning Algorithm of Underground Locomotive}

RSSI values show a great probability of randomness and volatility, when the wireless signal is propagating in coal mine. If only use formula (4) to calculate distance, $x_{\sigma}$ is unknown and it is not a solution. Without $x_{\sigma}$ or it takes 0 the fluctuation range of positioning error is large. Obviously this is not consistent with the positioning requirements of underground. To reduce the positioning error RSSI value correction is an essential part of the positioning algorithm. So a new correction model of RSSI value (Gaussian-weighted model) is proposed. Then estimate locomotive position using the least squares method. At last correct the estimation position using weighted distance correction.

3.1. Correct RSSI Value. Gaussian-weighted model combines the advantages of several models; concrete steps are as follows.

(1) High probability of RSSI values is selected by Gaussian model. When the unknown node receives several RSSI values from a same beacon node, the RSSI values with a probability density less than 0.6 (this value may be due to environment) are filtered by Gaussian model [13]. Arrange the remaining RSSI values from small to large: $\mathrm{RSSI}_{1} \leq \mathrm{RSSI}_{2} \leq \cdots \leq \mathrm{RSSI}_{m}$, where $m$ is number of remaining RSSI. 


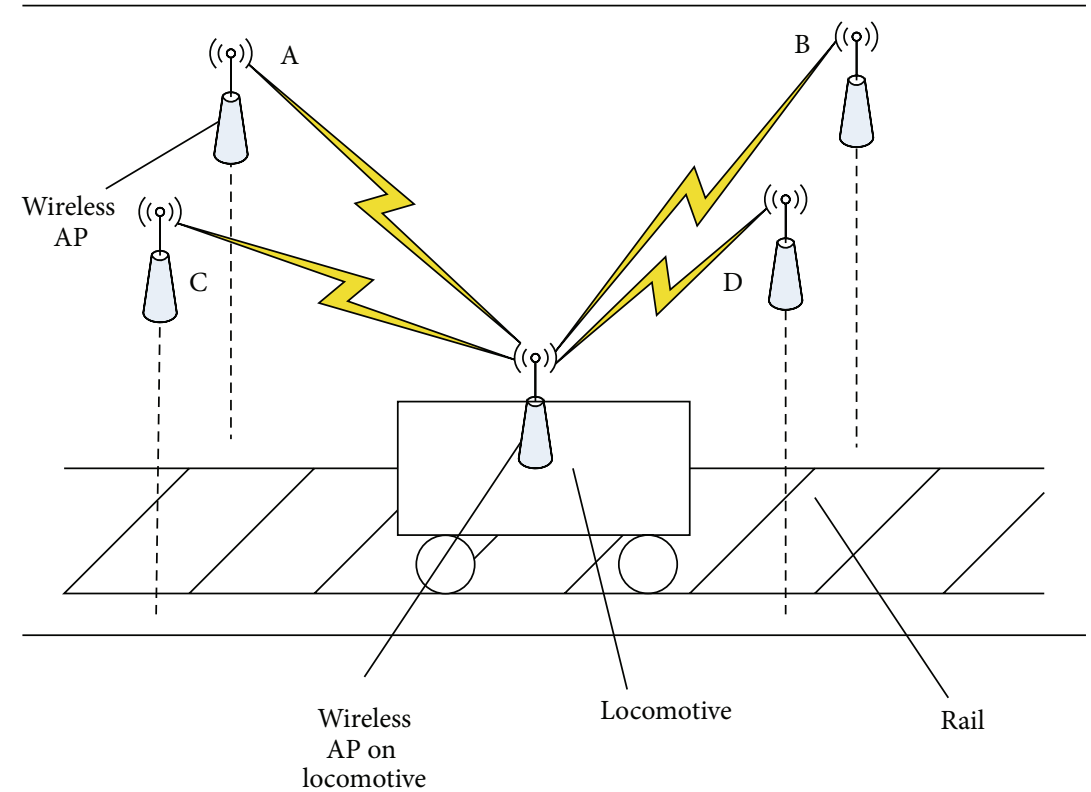

FIGURE 1: Wireless communication model.

(2) Calculate the weight of the RSSI values of $m$. Suppose that $\mu$ and $\sigma^{2}$ are the mean and variance of these RSSI values. Consider

$$
\begin{gathered}
\mu=\frac{1}{m} \sum_{i=1}^{m} \operatorname{RSSI}_{i}, \\
\sigma^{2}=\frac{1}{m} \sum_{i=1}^{m}\left(\operatorname{RSSI}_{i}-\mu\right)^{2} .
\end{gathered}
$$

Define the formula of weights:

$$
w_{i}=\frac{1 / \max \left\{\sigma^{2},\left(\mathrm{RSSI}_{i}-\mu\right)^{2}\right\}}{\sum_{i=1}^{m} 1 / \max \left\{\sigma^{2},\left(\mathrm{RSSI}_{i}-\mu\right)^{2}\right\}},
$$

where $\max \left\{\sigma^{2},\left(\operatorname{RSSI}_{i}-\mu\right)^{2}\right\}$ is to make the weight value smoother. As long as the difference between RSSI value and mean is less than the overall variance, take $\sigma^{2}$ to determine the weight of size. On the contrary, it is $\left(\mathrm{RSSI}_{i}-\mu\right)^{2}$. From (6) it can be drawn that the larger the difference, the smaller the weight.

(3) Calculate the RSSI value after correcting. Let the $m$ RSSI value multiply with the weight values of themselves; then sum the multiplied values as the correction RSSI value. The formula is

$$
\mathrm{RSSI}_{c}=\sum_{i=1}^{m} w_{i} \times \operatorname{RSSI}_{i}
$$

After correcting, formula (4) turns to

$$
d=10^{\left(\left(A-\mathrm{RSSI}_{c}\right) / 10 n\right)} d_{0} .
$$

3.2. Weighted Least Square Method. Suppose that the coordinate of locomotive is $A(x, y)$ and the coordinates of beacon nodes $A_{i}$ are $\left(x_{i}, y_{i}\right), i=1,2, \ldots, N$, where $N$ is the number of beacon nodes and $N \geq 4$. There are $N$ measurement equations, when each beacon node can communicate with the unknown node, which showed

$$
D_{i}=d_{i}+v_{i}, \quad i=1,2, \ldots, N,
$$

where $d_{i}=\sqrt{\left(x-x_{i}\right)^{2}+\left(y-y_{i}\right)^{2}}$ and $v_{i}$ is measurement error. Formula (9) is linearized and both sides are squared

$$
-2 x_{i} x-2 y_{i} y+x^{2}+y^{2}=\left(D_{i}-v_{i}\right)^{2}-\left(x_{i}^{2}+y_{i}^{2}\right) \text {. }
$$

Define $R$ and $R_{i}: R=\sqrt{x^{2}+y^{2}} ; R_{i}=\sqrt{x_{i}^{2}+y_{i}^{2}}$.

Formula (10) is turned to

$$
-2 x_{i} x-2 y_{i} y+R^{2}=D_{i}^{2}-R_{i}^{2}+v_{i}^{2}-2 D_{i} v_{i}
$$

Simplifying formula (11) we can obtain the following:

$$
\mathbf{h}=\mathbf{G} \boldsymbol{\theta}+\mathbf{v},
$$

where

$$
\boldsymbol{\theta}=\left[\begin{array}{lll}
x & y & R^{2}
\end{array}\right]^{T}, \quad \mathbf{h}=\left[\begin{array}{c}
D_{1}^{2}-R_{1}^{2} \\
D_{2}^{2}-R_{2}^{2} \\
\vdots \\
D_{N}^{2}-R_{N}^{2}
\end{array}\right]
$$




$$
\begin{gathered}
\mathbf{G}=\left[\begin{array}{ccc}
-2 x_{1} & -2 y_{1} & 1 \\
-2 x_{2} & -2 y_{2} & 1 \\
\vdots & \vdots & \vdots \\
-2 x_{N} & -2 y_{N} & 1
\end{array}\right], \\
\mathbf{v}=\left[\begin{array}{llll}
v_{1}^{2}-2 D_{1} v_{1} & v_{2}^{2}-2 D_{2} v_{2} & \cdots & v_{N}^{2}-2 D_{N} v_{N}
\end{array}\right]^{T} .
\end{gathered}
$$

The following is the least-square solution of formula (12):

$$
\boldsymbol{\theta}=\left(\mathbf{G}^{T} \mathbf{W G}\right)^{-1} \mathbf{G}^{T} \mathbf{W h}=\left[\begin{array}{lll}
\theta_{x} & \theta_{y} & \theta_{R^{2}}
\end{array}\right]^{T},
$$

where $\mathbf{W}$ is the inverse matrix of covariance matrix $\mathbf{v}$ of Gaussian noise.

3.3. Correct Positioning Error. Because weighted least square method cannot correct some positioning error which is caused by some inaccurate date, estimated location must make further correction and calculate more precise location. The method is weighted distance correction. Estimate the position of beacon nodes as unknown nodes and then the difference of coordinates between estimated position and actual position as the positioning errors and give them appropriate weight. Finally calculate correction value. There are 3 steps.

(1) Every beacon node is used as unknown node to estimate its own estimated position and calculate the error between estimated position and actual position. Suppose that the estimated position is $\left(x_{a i}, y_{a i}\right)$ which is calculated by the way of above. So the positioning error is

$$
e_{i}=\left[\begin{array}{c}
x_{i}-x_{a i} \\
y_{i}-y_{a i}
\end{array}\right] .
$$

(2) Determine the weights of localization errors of every beacon node. Because in the coal mine the closer the distance between beacon node and unknown node, the more similar of environment and the closer the positioning errors, its weight should be greater. The weights of $N$ beacon nodes which are involved in positioning are defined as follows:

$$
k_{i}=\frac{1 /\left(1+d_{i}\right)}{\sum_{i=1}^{N} 1 /\left(1+d_{i}\right)},
$$

where $d_{i}$ is the distance from unknown node to $N$ beacon nodes. They are calculated by formula (8).

(3) Calculate the final location coordinate. The correction values are obtained by multiplying formulae (15) and (16). Consider

$$
e=\left[\begin{array}{l}
e_{x}=\sum_{i=1}^{N} k_{i} \times\left(x_{i}-x_{a i}\right) \\
e_{y}=\sum_{i=1}^{N} k_{i} \times\left(y_{i}-y_{a i}\right)
\end{array}\right] .
$$

So the final positioning coordinates of unknown node (locomotive) are

$$
\begin{aligned}
& x=\theta_{x}+e_{x}, \\
& y=\theta_{y}+e_{y} .
\end{aligned}
$$

3.4. Select Better Beacon Nodes. Selected beacon node also has a great influence on the positioning error. A feature of the wireless signal is path loss when it is spread; the closer the distance between the node and locomotive, the smaller the path loss and the smaller the effect of various positioning error [16]. Equation (4) shows that the RSSI value is greater when it has closer communication distance. So the beacon nodes of bigger RSSI value are selected. These beacon nodes have more decision power to the location of the locomotive that can improve the positioning precision of the algorithm. The specific process is as follows.

(1) When the number of beacon nodes is larger or equal to 4 which is communication with the locomotive $N_{0} \geq 4$, arrange the RSSI values. Select ahead of $N$ $\left(4 \leq N \leq N_{0}\right)$ RSSI values as beacon nodes and involve in the calculation of the next step.

(2) When $N_{0}<4$, space some time and continue to signal until $N_{0} \geq 4$. Then execute step (1).

\section{Process of Improved Algorithm}

From the above analysis to improved algorithm for coal mine locomotive positioning, we can know that the process is divided into the following steps.

(1) Before receive positioning signal, beacon nodes calculate their own positioning errors using formula (15). Then they wait for positioning.

(2) After the positioning signal is emitted by locomotive, the nodes that can communicate with locomotive send the positioning errors which are calculated by step (1); then the locomotive records them. Do it repeatedly and record RSSI values. These exact RSSI values of each node can be calculated by formula (7).

(3) These RSSI values are arranged and select the number of N RSSI which is larger than others as beacon nodes.

(4) These RSSI values are translated into distance by formula (8). Then estimate the initial position of locomotive. At last determine the location of locomotive by formula (18).

\section{Simulation and Experiment}

Using MATLAB simulation platform for experiment, in order to make the simulation results more in line with the actual environment of locomotive, we selected a plane of $5 \mathrm{~m} \times$ $100 \mathrm{~m}$ and arranged nodes on both sides of the track. ZigBee technology is used as wireless communication technology. Because it shows low power consumption, low cost, short time delay, large network capacity, reliability, and security and 


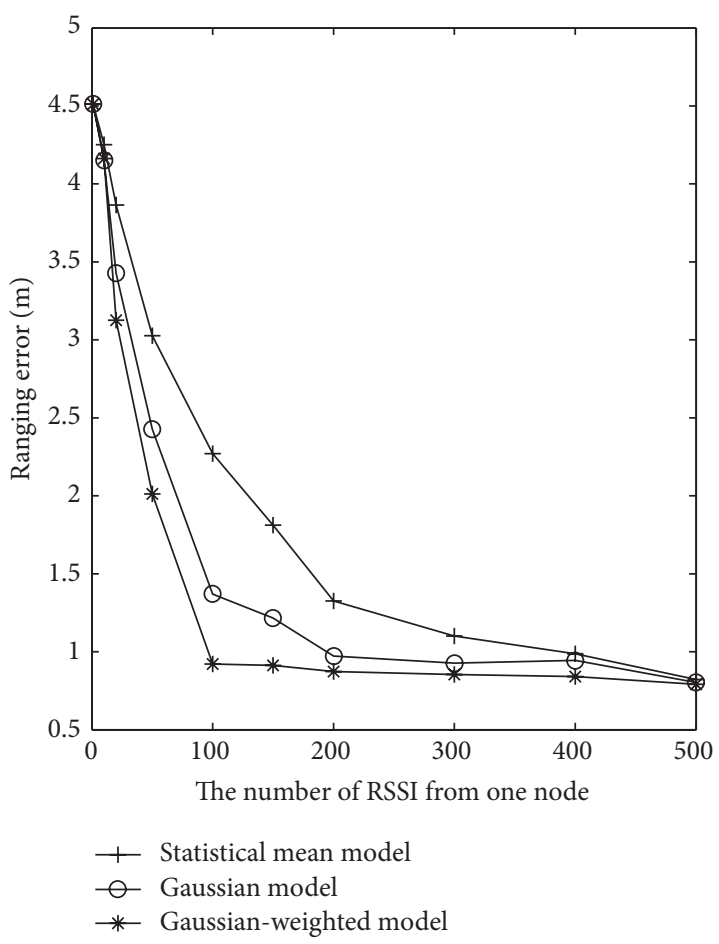

FIGURE 2: Relationship between the number of RSSI of a single node and ranging error.

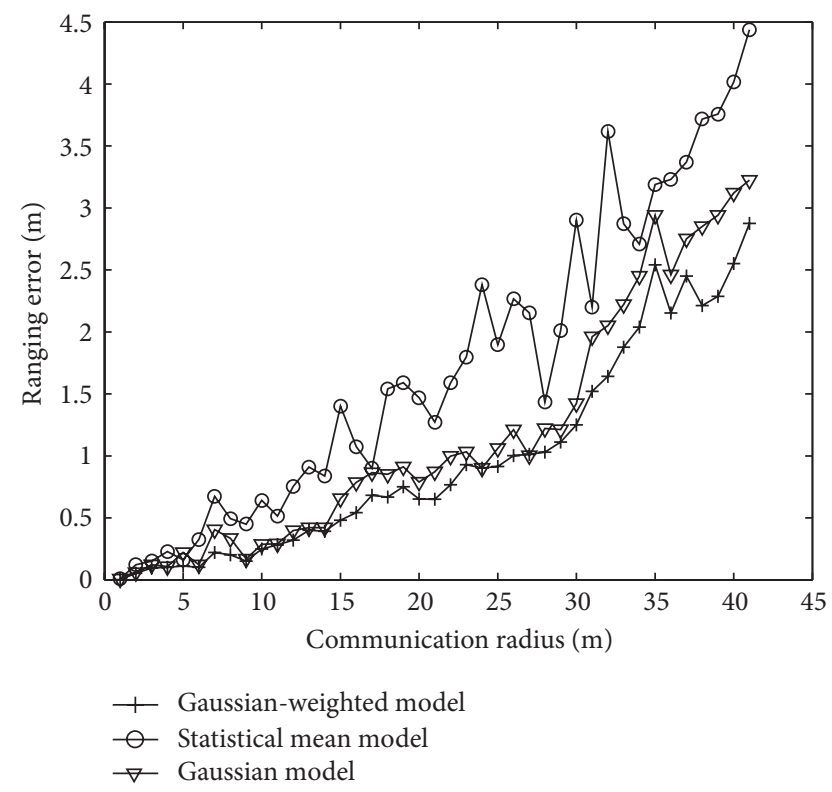

FIGURE 3: Comparison of the ranging error of RSSI.

can meet the need of mine. Suppose the initial coordinate of locomotive is $(0,0)$, path attenuation factor $n$ is $3.5, A$ takes $-45 \mathrm{~dB}, X_{\sigma}$ is Gaussian distribution whose mean is 0 , and standard deviation $\sigma$ is 6 . The speed of the locomotive is about $5 \mathrm{~m} / \mathrm{s}$. Six beacon nodes were in range of communication. Firstly, it will increase the communication and calculation
TABLE 1: Comparison of the ranging error.

\begin{tabular}{lccc}
\hline Error/m & $\begin{array}{c}\text { Statistical mean } \\
\text { model }\end{array}$ & $\begin{array}{c}\text { Gaussian } \\
\text { model }\end{array}$ & $\begin{array}{c}\text { Gaussian-weighted } \\
\text { model }\end{array}$ \\
\hline Average error & 2.2708 & 1.1689 & 0.9213 \\
Maximum error & 3.0328 & 1.8512 & 1.6789 \\
\hline
\end{tabular}

time of locomotive. Secondly, it will cause more interference. The simulation is divided into the following three aspects.

(1) Compare correction models of RSSI value. Compare ranging accuracy among statistical mean model, Gaussian model, and Gaussian-weighted model. If it has higher ranging accuracy, its correction of RSSI values is more precise. The result is shown in Figures 2 and 3.

Figure 2 is a relationship between the number of RSSI of a single node and ranging error and communication radius is $20 \mathrm{~m}$. The number of RSSI values of a single node has a great influence on ranging accuracy. When the number of RSSI value increases, the cost of the location is higher and the positioning accuracy increases. It can be seen from Figure 2 that the accuracy of the three models is increased, as the number of RSSI value increases, particularly within 100 the errors fall fastest. The Gaussian-weighted model has the highest ranging accuracy and the error decline is most obvious. When the number of RSSI values is more than 100, ranging error decreased slowly significantly and the positioning error of three models becomes closer. When the number of RSSI values is taken to 500 , ranging error is very similar. From the above analysis, it is best to take 100 as the number of the RSSI values in this simulation. That costs less and the Gaussian-weighted model maintains high ranging accuracy.

Figure 3 shows the comparison of ranging error of the three models. The number of the RSSI value of each node is 100. As can be seen from Figure 3, the error of statistical mean model is maximum and the maximum ranging error is $4.5 \mathrm{~m}$, approximating $10 \%$ of measurement distance. The error of Gaussianweighted model is minimum and the maximum ranging error is $3 \mathrm{~m}$, approximating $7 \%$ of measurement distance. With the expansion of communication radius, ranging errors of three models are increased. The error of statistical mean models fluctuates bigger and grows faster than the other two models. In order to obtain more accurate data, we took the communication radius $20 \mathrm{~m}$ and did experiment repeatedly to calculate the average error. The specific data are shown in Table 1.

The results can be taken from the experiment that the highest ranging precision is Gaussian-weighted model. Its average error is less than $1 \mathrm{~m}$ within communication radius of $20 \mathrm{~m}$ and maximum error does not exceed $2 \mathrm{~m}$. The lowest ranging precision 
TABLE 2: Comparison of the positioning error.

\begin{tabular}{|c|c|c|c|c|c|c|}
\hline Communication radius & \multicolumn{3}{|c|}{$5 \mathrm{~m}$} & \multicolumn{3}{|c|}{$10 \mathrm{~m}$} \\
\hline Ranging model & $\begin{array}{c}\text { Statistical } \\
\text { mean model }\end{array}$ & $\begin{array}{l}\text { Gaussian } \\
\text { model }\end{array}$ & $\begin{array}{c}\text { Gaussian-weighted } \\
\text { model }\end{array}$ & $\begin{array}{c}\text { Statistical } \\
\text { mean model }\end{array}$ & $\begin{array}{c}\text { Gaussian } \\
\text { model }\end{array}$ & $\begin{array}{c}\text { Gaussian-weighted } \\
\text { model }\end{array}$ \\
\hline $\begin{array}{l}\text { Positioning error } \\
\text { (without correction)/m }\end{array}$ & 0.4780 & 0.3526 & 0.3645 & 1.0838 & 1.0126 & 0.8213 \\
\hline $\begin{array}{l}\text { Positioning error } \\
\text { (correction) } / \mathrm{m}\end{array}$ & 0.4215 & 0.3215 & 0.3187 & 0.8838 & 0.6126 & 0.5213 \\
\hline Communication radius & \multicolumn{3}{|c|}{$20 \mathrm{~m}$} & \multicolumn{3}{|c|}{$40 \mathrm{~m}$} \\
\hline Ranging model & $\begin{array}{c}\text { Statistical } \\
\text { mean model }\end{array}$ & $\begin{array}{c}\text { Gaussian } \\
\text { model }\end{array}$ & $\begin{array}{c}\text { Gaussian-weighted } \\
\text { model }\end{array}$ & $\begin{array}{c}\text { Statistical } \\
\text { mean model }\end{array}$ & $\begin{array}{c}\text { Gaussian } \\
\text { model }\end{array}$ & $\begin{array}{c}\text { Gaussian-weighted } \\
\text { model }\end{array}$ \\
\hline $\begin{array}{l}\text { Positioning error } \\
\text { (without correction)/m }\end{array}$ & 1.9421 & 1.5765 & 1.3569 & 5.3751 & 3.4548 & 3.0521 \\
\hline $\begin{array}{l}\text { Positioning error } \\
\text { (correction) } / \mathrm{m}\end{array}$ & 1.6421 & 1.1765 & 0.9569 & 4.3751 & 2.7548 & 2.0521 \\
\hline
\end{tabular}

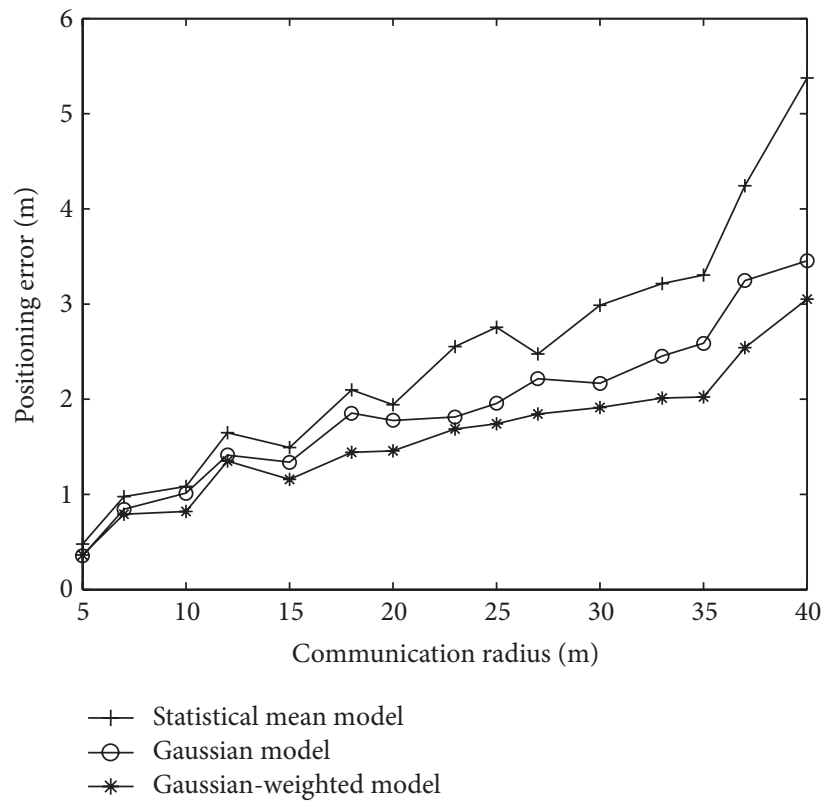

(a) Without positioning error correction

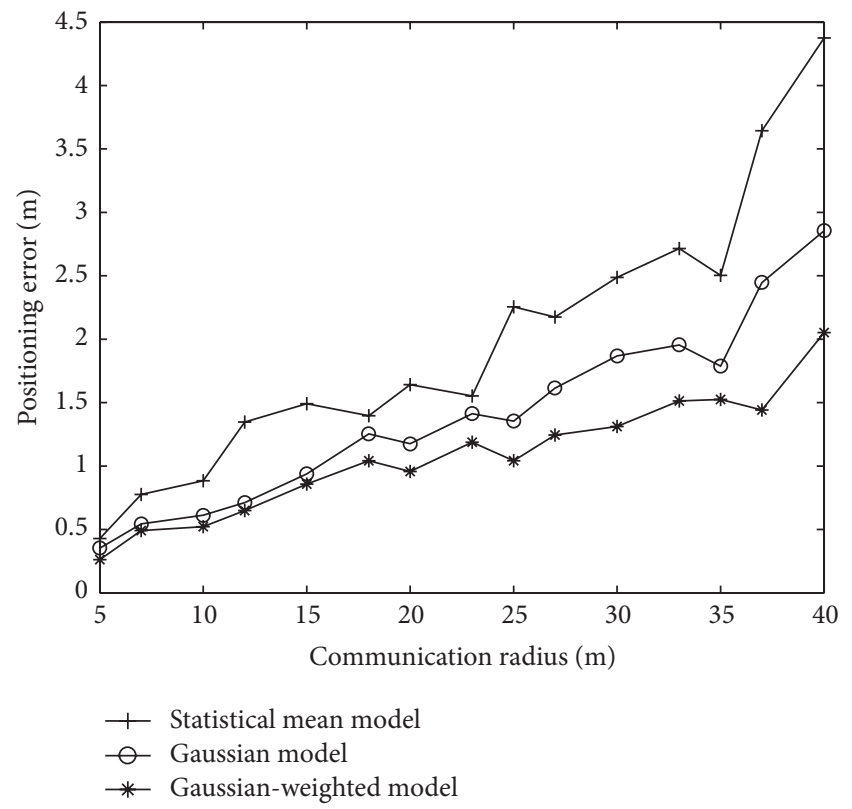

(b) With positioning error correction

FIGURE 4: Comparison of these positioning algorithms with or without positioning error correction.

is statistical mean model. Its average ranging error already exceeds $2 \mathrm{~m}$ within the communication radius of $20 \mathrm{~m}$. Gaussian model is between them; compared to the statistical mean model the error is more stable. So the most accurate correction model of RSSI value is Gaussian-weighted model and its error is more stable.

(2) Comparison of positioning error. Firstly, compare positioning accuracy of statistical mean model, Gaussian model, and Gaussian-weighted models with or without weighted distance correction. The result is shown in Figure 4. Secondly, the positioning accuracies are compared with different numbers of the beacon nodes. The result is shown in Table 3.

From Figure 4(a) it can be seen that the minimum positioning error is Gaussian-weighted model and with the increasing of communication radius it grows more stable. The maximum positioning error is statistical mean model and the error is not stable enough. Gaussian model is between the other two models and the error grows stable.

Comparing the positioning error of Figures 4(a) and 4(b), it can be seen that if algorithm is corrected by weighted distance correction, it is more accurate than without correcting. Gaussian-weighted model is the best. At a communication radius of $40 \mathrm{~m}$, error of Gaussian-weighted model is reduced $33 \%$, while the statistical mean model and the Gaussian model are reduced $19 \%$ and $22 \%$. The specific comparison data is shown in Table 2. 
TABLE 3: Comparison of the positioning error with different beacon nodes.

\begin{tabular}{lcccc}
\hline The size of $N$ & 4 & 6 & 8 & 10 \\
\hline Statistical mean model & 1.7708 & 16589 & 1.5913 & 1.5237 \\
Gaussian model & 1.4028 & 1.2012 & 1.1189 & 1.0756 \\
Gaussian-weighted model & 1.2617 & 0.9636 & 0.9337 & 0.9165 \\
\hline
\end{tabular}

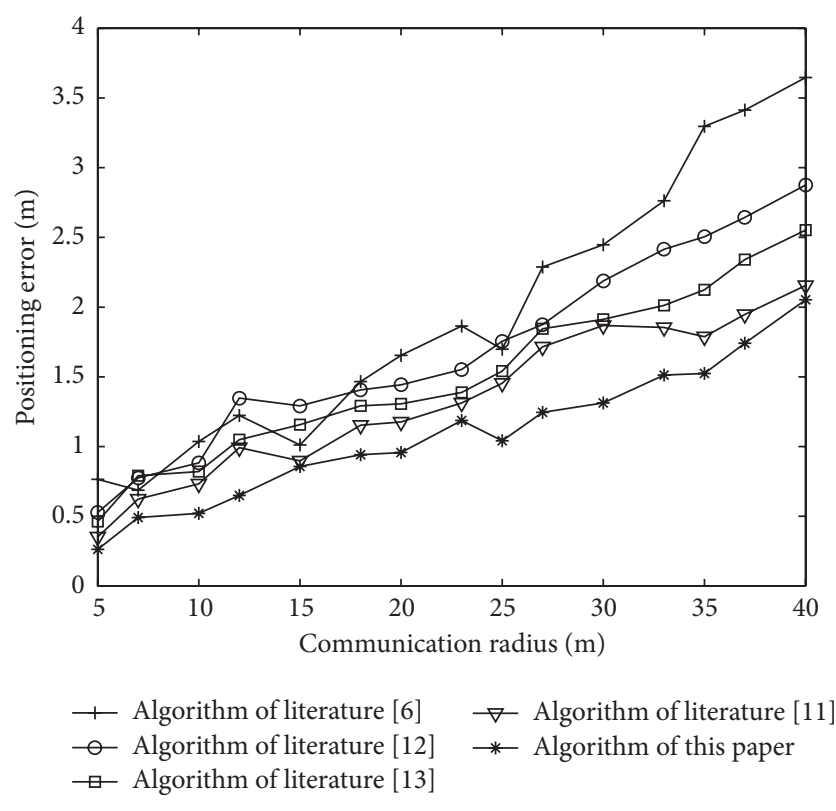

FIgURE 5: Comparison of the positioning error among different algorithms.

In Table 3, communication radius is $20 \mathrm{~m}$. From Table 3 it can be seen that positioning error is small when the number of beacon nodes increased. But with the increase of beacon nodes, the reduced amount of positioning error is decreased, particularly Gaussianweighted model. This is because the amount of calculation of Gaussian-weighted model is larger than the Gaussian model and the statistical mean model. It will cost more time to perform the calculation when the beacon nodes are increased. So positioning error will become large when the locomotive is moving. From Table 3 and considering the cost, 6 is the most appropriate choice.

(3) For comparison of positioning algorithm, compare these algorithms of literatures $[6,11-13]$ and this paper in the same environment; the simulation result is shown in Figure 5. As can be seen from Figure 5, the positioning accuracy of this paper is highest. The algorithm of literature [6] has high positioning accuracy within $25 \mathrm{~m}$. Out of $25 \mathrm{~m}$, its positioning grows faster than others. The other three algorithms have higher positioning error than this paper, but they are more stable than in the literature [6].

The results of above experiment show that in the case of positioning error correction Gaussian-weighted model has highest positioning accuracy. The smaller the communication radius, the less the positioning error is. When the communication radius is $10 \mathrm{~m}$, the positioning accuracy can be maintained at about $0.5 \mathrm{~m}$.

\section{Conclusions}

(1) This paper analyzes a number of factors of locomotive positioning errors for coal mine and proposes a coal mine locomotive positioning algorithm based on RSSI. Because the RSSI value in coal mine is very unstable, it is corrected by weighted-Gaussian model. This model combines the advantages of Gaussian model and weighted model. It has high ranging precision in the coal mine of harsh environment.

(2) Just using weighted least square method to estimate the position of locomotive cannot reduce the impact of some inaccuracy dates effectively. Correcting the estimated position is necessary. This paper proposes a weighted distance correction method. The positioning errors of each beacon node are calculated using Gaussian-weighted model and weighted according to the distances from locomotive to the beacon node. The closer the distance is, the greater the weight is.

(3) The algorithm which is proposed is simulated by MATLAB. The results of experiment show that Gaussian-weighted model is the best model. In the case of positioning error correction, positioning error is descendant while communication radius is the same and the minimum positioning error is less than $0.5 \mathrm{~m}$.

\section{Conflict of Interests}

The authors declare that there is no conflict of interests regarding the publication of this paper.

\section{Acknowledgments}

This work was financially supported by the National Natural Science Foundation of China (61070220 and 61170060), Anhui Provincial Natural Science Foundation of China (1408085ME110), Anhui Provincial Major Project of Colleges and Universities Natural Science (KJ2013ZD09), and Anhui Provincial Key Project of Colleagues and Universities Natural Science (KJ2012A096).

\section{References}

[1] M. D. Bedford and G. A. Kennedy, "Evaluation of ZigBee (IEEE 802.15.4) time-of-flight-based distance measurement for application in emergency underground navigation," IEEE Transactions on Antennas and Propagation, vol. 60, no. 5, pp. 2502-2510, 2012

[2] M. Lie, D. Enjie, F. Qiyan et al., "Underground moving target positioning and historical trajectory extraction based on Wi-Fi 
and WebGI," Geography and Geo-Information Science, vol. 28, no. 3, pp. 109-110, 2013.

[3] H. Chi, K. Zhan, and B. Shi, "Automatic guidance of underground mining vehicles using laser sensors," Tunnelling and Underground Space Technology, vol. 27, no. 1, pp. 142-148, 2012.

[4] Y. Gao, H. Sun, and Y. Yang, "The locomotive position system in coal pit based on the piezoelectric accelerometer," Piezoelectrics and Acoustooptics, vol. 34, no. 5, pp. 782-784, 2012.

[5] Q.-Z. Zhang, S.-B. Zhang, J. Wang, and H.-F. Bian, "Lowcost GPS/INS in-motion alignment model for open-pit mine transport truck monitoring and dispatch system," Journal of the China Coal Society, vol. 38, no. 8, pp. 1362-1367, 2013.

[6] D.-S. Han, W. Yang, Y. Liu, and Y. Zhang, "A weighted centroid localization algorithm based on received signal strength indicator for underground coal mine," Journal of the China Coal Society, vol. 38, no. 3, pp. 522-528, 2013.

[7] X. Wang, S. Yuan, R. Laur, and W. Lang, "Dynamic localization based on spatial reasoning with RSSI in wireless sensor networks for transport logistics," Sensors and Actuators, A: Physical, vol. 171, no. 2, pp. 421-428, 2011.

[8] R.-B. Zhang, J.-G. Guo, F.-H. Chu, and Y.-C. Zhang, "Environmental-adaptive indoor radio path loss model for wireless sensor networks localization," International Journal of Electronics and Communications, vol. 65, no. 12, pp. 1023-1031, 2011.

[9] Z. Wei, Z. Lv, C. Yang, J. Han, and L. Shi, "Wireless positioning method based on correctness judgment of received signal strength for mine locomotive," Chinese Journal of Scientific Instrument, vol. 35, no. 1, pp. 178-184, 2014.

[10] J.-W. Zhang, L. Zhang, Y. Ying, and F. Gao, "Research on distance measurement based on RSSI of ZigBee," Chinese Journal of Sensors and Actuators, vol. 22, no. 2, pp. 285-288, 2009.

[11] G. Wan, J. Zhong, and C. Yang, "Improved algorithm of ranging and locating based on RSSI," Application Research of Computers, vol. 29, no. 11, pp. 4156-4158, 2012.

[12] J.-Q. Xu, W. Liu, Y.-Y. Zhang, and C.-L. Wang, "RSSI-based antiinterference WSN positioning algorithm," Journal of Northeastern University, vol. 31, no. 5, pp. 647-650, 2010.

[13] W. Tao, Y. Zhu, and Z. Jia, "A distance measurement algorithm based on RSSI hybrid filter and least square estimation," Chinese Journal of Sensors and Actuators, vol. 25, no. 12, pp. 1748-1753, 2012.

[14] J. Zheng, C. Wu, H. Chu, and Y. Xu, "An improved RSSI measurement in wireless sensor networks," Procedia Engineering, vol. 15, pp. 876-880, 2011.

[15] G. Blumrosen, B. Hod, T. Anker, D. Dolev, and B. Rubinsky, "Enhanced calibration technique for RSSI-based ranging in body area networks," Ad Hoc Networks, vol. 11, no. 1, pp. 555569, 2013.

[16] X. Luo, W. J. O’Brien, and C. L. Julien, “Comparative evaluation of received signal-strength index (RSSI) based indoor localization techniques for construction jobsites," Advanced Engineering Informatics, vol. 25, no. 2, pp. 355-363, 2011. 

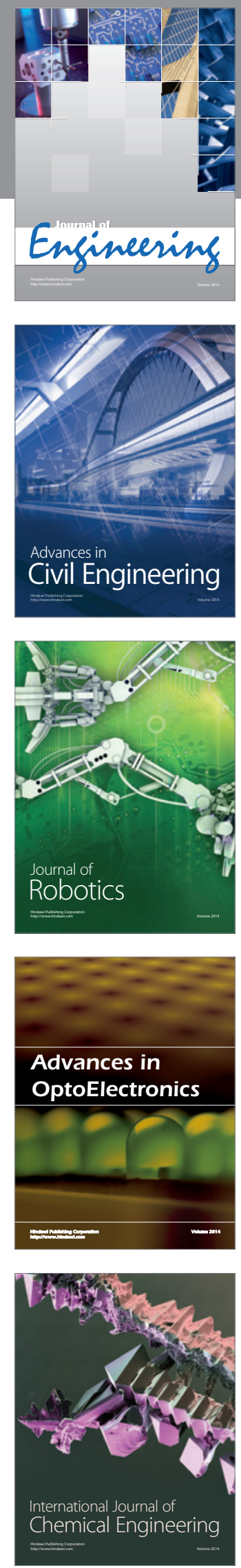

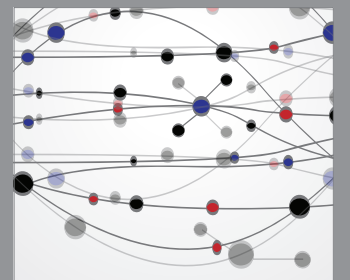

The Scientific World Journal
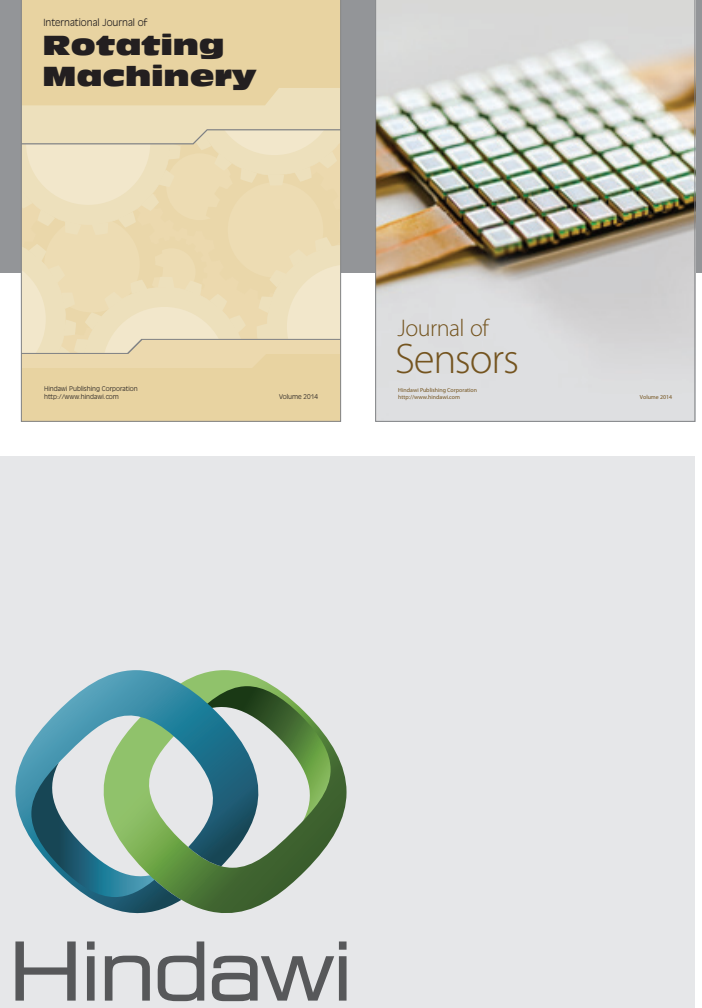

Submit your manuscripts at http://www.hindawi.com
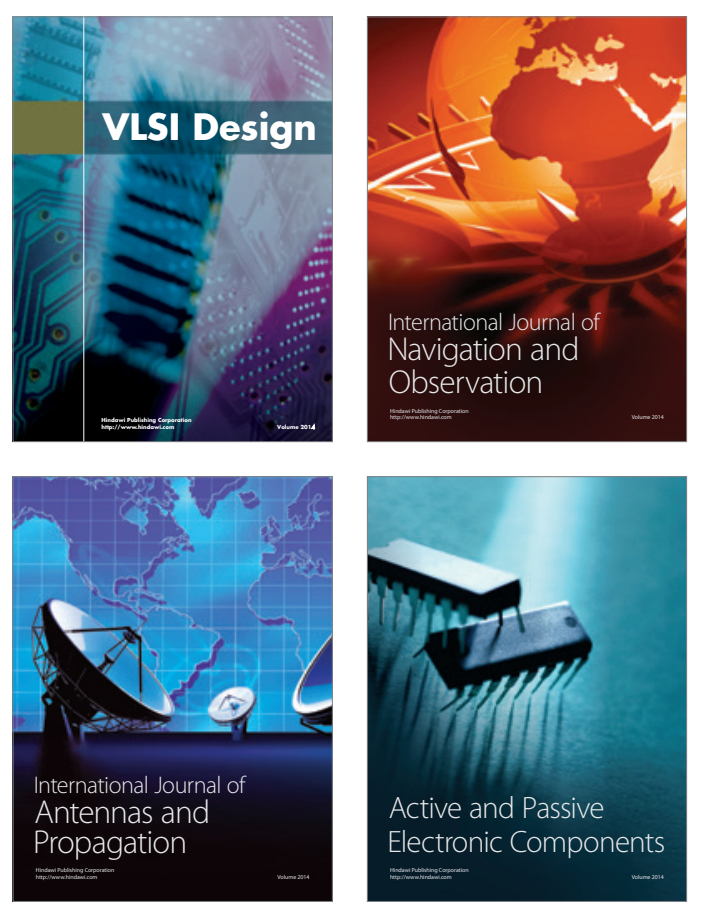
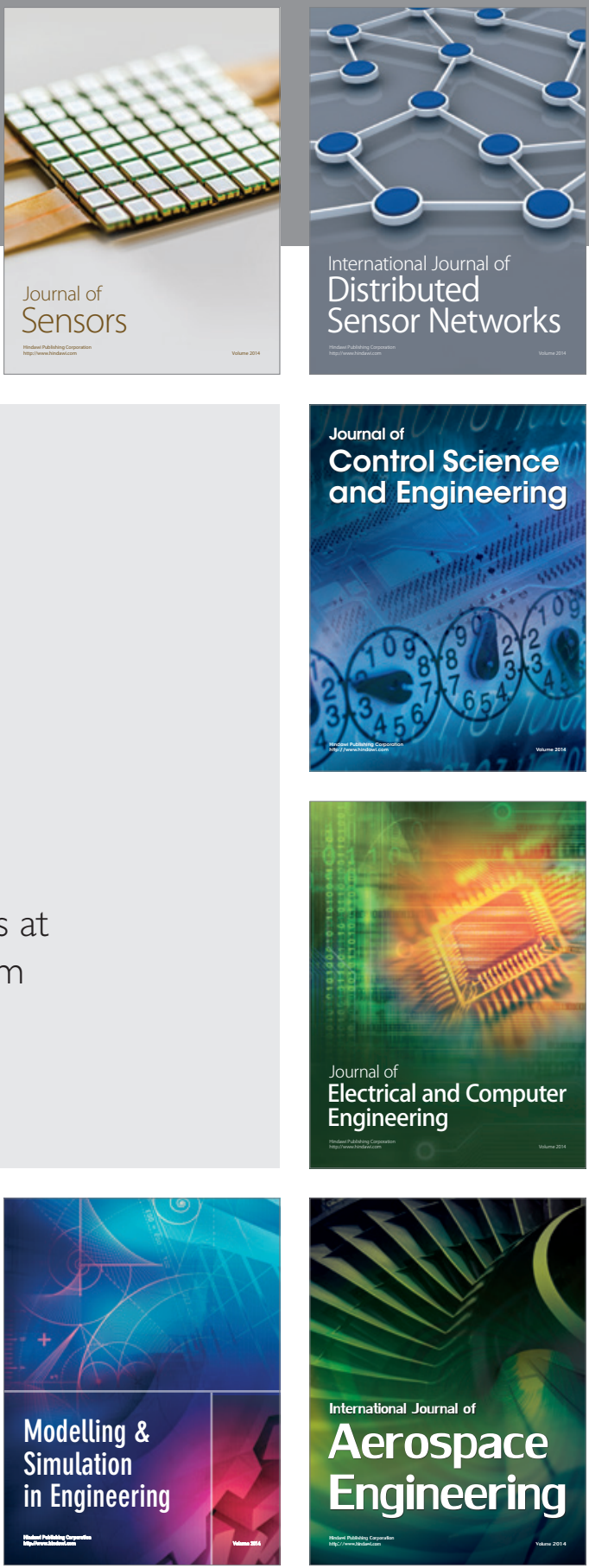

Journal of

Control Science

and Engineering
